\title{
Numerical aspects of finite element simulations of residual stresses in metal matrix composites
}

\author{
F. Teixeira-Dias ${ }^{1, *, \dagger}$ and L. F. Menezes ${ }^{2}$ \\ ${ }^{1}$ Department of Mechanical Engineering, University of Aveiro, Campus de Santiago, 3810-193 AVEIRO, Portugal \\ ${ }^{2}$ Department of Mechanical Engineering - CEMUC, University of Coimbra, 3030 COIMBRA, Portugal
}

\begin{abstract}
SUMMARY
When a metal matrix composite (MMC) is cooled down from the fabrication or annealing temperature to room temperature, residual stresses are induced in the composite due to the mismatch of the thermal expansion coefficients of the matrix and reinforcement. A thermomechanical model describing these processes is presented considering that the reinforcement component has a thermo-elastic behaviour and that the matrix material exhibits a thermo-elastoviscoplastic behaviour. The model is implemented with a semi-implicit forward gradient finite element method algorithm and the resulting code is used to perform numerical simulations and calculate thermally induced residual stress fields in MMCs. Several tests are performed on a continuously reinforced MMC and a short cylindrical particle MMC in order to optimize the algorithm and define its governing parameters. Good agreement was obtained with results from other authors. Copyright (c) 2001 John Wiley \& Sons, Ltd.
\end{abstract}

KEY WORDS: metal matrix composites; residual stresses; numerical simulation; optimization algorithm; material behaviour

\section{INTRODUCTION}

Metal matrix composites (MMC) have become increasingly attractive in recent years for their high strength and creep resistance properties [1-9]. When cooling down a metal matrix composite from the fabrication temperature to the room temperature, thermally induced residual stresses are generated. The development of residual stresses and the mechanical behaviour of MMCs in the presence of these stresses has been thoroughly studied by analytical, numerical or experimental methods (e.g. References [1-3, 8-17]).

Some specific theoretical and numerical models have been proposed in order to evaluate these residual stresses. Both Arsenault and Taya [16] and Withers et al. [18] used Eshelby's equivalent inclusion approach to provide a theoretical basis for the prediction of the mechanical properties

\footnotetext{
*Correspondence to: F. Teixeira-Dias, Department of Mechanical Engineering, University of Aveiro, Campus de Santiago, 3810-193 Aveiro, Portugal

†E-mail: ftd@mec.ua.pt

Contract/grant sponsor: FCT (Portuguese Science and Technology Foundation)
}

Copyright (c) 2001 John Wiley \& Sons, Ltd.

Received 4 June 1999

Revised 15 February 2000 
of short fibre metal matrix composites. This approach allows the prediction of thermal residual stresses in MMCs considering thermoelastic fibres and plastic matrixes. Nevertheless it has a drawback of being limited in the fields of the geometric and physical modelation of the problem. Dunn and Taya [19] also proposed an analysis of thermally induced residual stresses in metal matrix composites based on Eshelby's theory. The former studies the interaction among fibres at finite-volume fractions through the Mori-Tanaka mean field theory and treats the matrix as an elastic/plastic material while the reinforcement is considered elastic. Povirk et al. [1] presented a general formulation valid for finite strains and rotations where account was taken of thermoelasticity in the reinforcement and of temperature-dependent plasticity in the matrix. The flow theory of plasticity was used by Zahl et al. [8] to characterize a rate-independent ductile matrix while the reinforcement was considered both brittle and elastic. A mathematically more elaborate constitutive model was presented by Zywickz and Parks [12]. In this model the matrix material parameters are temperature-dependent, including the coefficient of thermal expansion (CTE). The model used can describe a temperature- and time-dependent inelastic and isotropic matrix deformation, while the fibre reinforcements are modelled as linearly elastic and transversely isotropic. Suéry et al. [9] and Teodosiu and Menezes [10] studied the residual stress fields in an aluminium matrix with a spherical SiC particle. For this particular purpose a one-dimensional axisymmetric finite element code was developed considering a thermo-elastoviscoplastic behaviour for the Al matrix and a thermo-elastic behaviour for the $\mathrm{SiC}$ reinforcement.

More recent works have been published in this domain, but most of them present models that are only one or two dimensional. This fact is an obvious limitation to the study of complex and more general reinforcement geometries.

The purpose of this paper is to present a fully three-dimensional finite element algorithm dedicated to the numerical calculation of thermaly induced residual stresses in metal matrix composite materials. The mechanical model considers the reinforcement component to behave thermoelastically and the matrix material to have a thermo-elastoviscoplastic behaviour. The developed code is tested with some numerical examples concerning a $\mathrm{SiC}$ reinforcement in an aluminium matrix in order to validate and optimize the numerical algorithms that were implemented.

\section{THERMO-MECHANICAL MODEL}

The temperature field among the composite is assumed to be homogeneous at all times and its evolution given by

$$
T(t)=T_{0}+\dot{T} t
$$

where $t$ is the time, $T_{0}$ is the initial fabrication temperature at time $t=t_{0}$ and $\dot{T}$ the constant cooling rate.

Since the maximum temperature reached by the reinforcement of the MMC is significantly apart from its decomposition temperature, it is assumed that the reinforcement behaves in a thermoelastic manner. On the other hand, the homologous temperature of the metallic matrix material varies from values close to 1 , at $t=0$, to approximately 0.3 on cooling down to room temperature. Thus, the inelastic properties of the matrix material must be accounted for over the whole temperature range. It is then assumed that the matrix material has a thermo-elastoviscoplastic behaviour. This behaviour will be described by the stress tensor $\boldsymbol{\sigma}$, the temperature field $T(t)$ and a structural scalar 
parameter $s$ that accounts for the isotropic work-hardening and recovery phenomena happening on the matrix material.

\subsection{Constitutive relations for the matrix}

To specify the constitutive relations of the matrix material it is first assumed that the total strain rate tensor $\mathbf{D}$ is decomposed as

$$
\mathbf{D}=\mathbf{D}^{\mathrm{e}}+\mathbf{D}^{\text {th }}+\mathbf{D}^{\mathrm{p}}
$$

where $\mathbf{D}^{\mathrm{e}}, \mathbf{D}^{\text {th }}=\alpha_{\mathrm{M}} \dot{T} \mathbf{1}$ and $\mathbf{D}^{\mathrm{p}}$ are the elastic, thermal and viscoplastic strain rate tensors, respectively. $\alpha_{M}$ is the thermal expansion coefficient of the matrix material and $\mathbf{1}$ is the second-order identity tensor. The viscoplastic deformation is assumed to be isochoric, i.e. $\operatorname{tr} \mathbf{D}^{\mathrm{p}}=0$.

Most of the thermo-elastoviscoplastic mechanical models assume that the material is isotropic and that the plastic strain rate depends on the stress state, the temperature and a state variable representing the strain history of the material (e.g. References [20-23]). Based on this assumption, the viscoplastic strain rate tensor is given by

$$
\mathbf{D}^{\mathrm{p}}=\frac{3 \boldsymbol{\sigma}^{\prime}}{2 \bar{\sigma}} \dot{\bar{\varepsilon}}^{\mathrm{p}}
$$

where $\boldsymbol{\sigma}^{\prime}$ is Cauchy's deviatoric stress tensor, $\bar{\sigma}$ is the equivalent stress

$$
\bar{\sigma}=\left(\frac{3}{2} \boldsymbol{\sigma}^{\prime}: \boldsymbol{\sigma}^{\prime}\right)^{1 / 2}
$$

and $\dot{\bar{\varepsilon}}^{\mathrm{p}}=f(\bar{\sigma} ; s ; T)$ is the equivalent plastic strain rate. $T=T(t)$ is the temperature and $s$ the scalar parameter that accounts for the microstructural state of the material, its evolution being itself a function of $\bar{\sigma}, s$ and $T$ :

$$
\dot{s}=g(\bar{\sigma} ; s ; T)=\dot{\bar{\varepsilon}}^{\mathrm{p}} h(\bar{\sigma} ; s ; T)
$$

After the experimental tests done by Anand [21], it can be shown that the functional dependence between strain rate and stress depends itself on the material. The data from these tests suggest that the behaviour of 1100 -aluminium can be correctly represented by a power law while iron - 2 per cent silicon is better represented by an exponential dependence. In order to accommodate both dependencies of the strain rate on the stress, the adopted specific form for function $f$ was [22]

$$
f(\bar{\sigma} ; s ; T)=A \exp \left(-\frac{Q}{R_{g} T}\right)\left[\sinh \left(\xi \frac{\bar{\sigma}}{s}\right)\right]^{1 / m}
$$

Thus, function $h(\bar{\sigma} ; s ; T)$ that represents the hardening behaviour becomes

$$
h(\bar{\sigma} ; s ; T)=h_{0}\left|1-\frac{s}{s^{*}}\right|^{a} \operatorname{sign}\left(1-\frac{s}{s^{*}}\right)
$$

with $a \geqslant 1$, which is a simple modification of the function proposed by Anand [21], who only considered the particular case of $a=1 . h_{0}$ is the constant rate of athermic hardening. The quantity $s^{*}$ is a saturation value of $s$, associated with a given temperature/strain rate pair and is taken to be given by

$$
s^{*}=\bar{s}\left[\frac{\dot{\bar{\varepsilon}}^{\mathrm{p}}}{A} \exp \left(\frac{Q}{R_{\mathrm{g}} T}\right)\right]^{n}
$$


In Equation (8) $A, Q, \xi, m, h_{0}, a, \bar{s}$ and $n$ are material parameters. In Equation (7), $|x|$ and $\operatorname{sign}(x)$ denote the absolute value and sign of $x$, respectively. These mathematical operators are introduced to allow the accommodation of situations where the current value of $s$ is greater than its saturation value, given by Equation (8). This allows the modelling of strain hardening and also strain softening situations. Such a situation is certainly possible in hot working processes where the strain rate decreases or the temperature increases at a given material point. Such changes may cause the current value of $s$ to be greater than the asymptotic value represented by $s^{*}$, calculated under the new conditions [22].

\subsection{Constitutive relations for the reinforcement}

As mentioned above, the reinforcement of the metal matrix composite material is considered to behave thermoelastically. Thus its constitutive relations are simply given by

$$
\dot{\boldsymbol{\sigma}}=\mathbf{C}^{\mathrm{e}}: \mathbf{D}^{\mathrm{e}}-3 K_{\mathrm{R}} \alpha_{\mathrm{R}} \dot{T} \mathbf{1}
$$

In the previous relation $\mathbf{C}^{\mathrm{e}}$ is the elastic modulus of the reinforcement material and $K_{\mathrm{R}}$ and $\alpha_{\mathrm{R}}$ are the bulk modulus and the thermal expansion coefficient for the reinforcement, respectively.

\section{TIME INTEGRATION PROCEDURE}

An incremental time integration procedure is implemented where the state variables $\boldsymbol{\sigma}_{n}, s_{n}$ and $T_{n}$ are assumed to be known at time instant $t_{n}$. The evolution equation must then be integrated over the time increment $\Delta t$ in order to calculate the state variables $\boldsymbol{\sigma}_{n+1}, s_{n+1}$ and $T_{n+1}$ at time instant $t_{n+1}=t_{n}+\Delta t$.

\subsection{Forward gradient}

A forward gradient integration procedure is implemented based on an estimation of the integral of $\mathbf{D}^{\mathrm{p}}$ over the current time increment $\Delta t$, in order to obtain the plastic strain increment $\Delta \boldsymbol{\varepsilon}^{\mathrm{p}}$. The increment of the deviatoric Cauchy stress may be expressed as

$$
\Delta \boldsymbol{\sigma}^{\prime}=2 \mu_{\mathrm{M}}\left(\Delta \boldsymbol{\varepsilon}^{\prime}-\Delta \boldsymbol{\varepsilon}^{\mathrm{p}}\right)
$$

where the plastic strain increment is given by

$$
\Delta \boldsymbol{\varepsilon}^{\mathrm{p}}=\int_{t_{n}}^{t_{n+1}} \mathbf{D}^{\mathrm{p}} \mathrm{d} t
$$

The total strain increment and its deviatoric part are, respectively,

$$
\Delta \boldsymbol{\varepsilon}=\int_{t_{n}}^{t_{n+1}} \mathbf{D} \mathrm{d} t
$$

and $\Delta \boldsymbol{\varepsilon}^{\prime}=\Delta \boldsymbol{\varepsilon}-(1 / 3) \operatorname{tr}(\Delta \boldsymbol{\varepsilon}) \mathbf{1}$. An estimate for the plastic strain rate increment of Equation (11) is obtained with the generalized mid-point rule:

$$
\Delta \boldsymbol{\varepsilon}^{\mathrm{p}}=\left[\mathbf{D}_{n}^{\mathrm{p}}+\Phi\left(\mathbf{D}_{n+1}^{\mathrm{p}}-\mathbf{D}_{n}^{\mathrm{p}}\right)\right] \Delta t
$$


where $0 \leqslant \Phi \leqslant 1[9,10,24,25]$. After some intermediate calculation and using a truncated Taylor expansion of $\mathbf{D}^{\mathrm{p}}$ around $t_{n}$ to approximate $\mathbf{D}_{n+1}^{\mathrm{p}}$, Equation (13) becomes

$$
\Delta \boldsymbol{\varepsilon}^{\mathrm{p}}=\Delta \bar{\varepsilon}^{\mathrm{p}}\left(\frac{3 \boldsymbol{\sigma}_{n}^{\prime}}{2 \bar{\sigma}_{n}}\right)+\frac{3}{2 h_{1}}\left[\Delta \boldsymbol{\sigma}^{\prime}-\left(\boldsymbol{\sigma}_{n}^{\prime}: \Delta \boldsymbol{\sigma}^{\prime}\right)\left(\frac{3 \boldsymbol{\sigma}_{n}^{\prime}}{2 \bar{\sigma}_{n}^{2}}\right)\right]
$$

with $h_{1}=\bar{\sigma}_{n} /\left(\Phi f_{n} \Delta t\right)$ and

$$
\Delta \bar{\varepsilon}^{\mathrm{p}}=\left[f_{n}+\Phi\left(\frac{\partial f_{n}}{\partial \bar{\sigma}} \Delta \bar{\sigma}+\frac{\partial f_{n}}{\partial s} \Delta s+\frac{\partial f_{n}}{\partial T} \Delta T\right)\right] \Delta t
$$

Equation (15) can be solved after the substitution of $\Delta \bar{\sigma}$ and $\Delta s$ by their estimates

$$
\Delta \bar{\sigma}=\frac{3 \mu_{\mathrm{M}}}{\bar{\sigma}_{n}} \boldsymbol{\sigma}_{n}: \Delta \boldsymbol{\varepsilon}-3 \mu_{\mathrm{M}} \Delta \bar{\varepsilon}^{\mathrm{p}}
$$

and

$$
\Delta s=\Delta \bar{\varepsilon}^{\mathrm{p}} h(\bar{\sigma} ; s ; T) .
$$

Using Equations (10), (14) and (15) the increment of the Cauchy stress tensor becomes

$$
\Delta \boldsymbol{\sigma}=\mathbf{L}^{\tan }: \Delta \boldsymbol{\varepsilon}-K_{2} \boldsymbol{\sigma}_{n}^{\prime}-3 K_{\mathrm{M}} \alpha_{\mathrm{M}}(\Delta T) \mathbf{1}
$$

where $\mathbf{L}^{\tan }$ is the tangent elastoviscoplastic modulus whose components are given by

$$
L_{i j k l}^{\mathrm{tan}}=\bar{\lambda}_{n} \delta_{i j} \delta_{k l}+\bar{\mu}_{n}\left(\delta_{i k} \delta_{j l}+\delta_{i l} \delta_{j k}\right)-K_{1} \sigma_{i j}^{\prime} \Delta \sigma_{k l}^{\prime}
$$

where $\delta_{i j}$ is the delta of Kronecker and

$$
\begin{aligned}
K_{1} & =\frac{3}{\bar{\sigma}_{n}^{2}}\left[\frac{v_{n}}{1+v_{n}} \frac{3 \mu_{\mathrm{M}}^{2}}{G_{n}}-\left(\mu_{\mathrm{M}}-\bar{\mu}_{n}\right)\right] \\
v_{n} & =\Phi \frac{\partial f_{n}}{\partial \bar{\sigma}} G_{n} \Delta t \\
\bar{\lambda}_{n} & =K_{M}-\frac{2}{3} \bar{\mu}_{n} \\
\bar{\mu}_{n} & =\frac{\mu_{\mathrm{M}}}{1+3 \mu_{M} / h_{1}} \\
G_{n} & =3 \mu_{M}-\left(\frac{\partial f_{n}}{\partial s} / \frac{\partial f_{n}}{\partial \bar{\sigma}}\right) h\left(\bar{\sigma}_{n} ; s_{n} ; T_{n}\right) \\
K_{2} & =\frac{\Delta t}{1+v_{n}}\left(f_{n}+\frac{\partial f_{n}}{\partial T} \Phi \Delta T\right) \frac{3 \mu_{\mathrm{M}}}{\bar{\sigma}_{n}}
\end{aligned}
$$

\section{NUMERICAL IMPLEMENTATION AND ALGORITHM}

\subsection{Virtual work principle}

For the thermo-mechanical model described above the Virtual Work Principle can be written as [26]

$$
\int_{V} \sigma_{i j} \delta \varepsilon_{i j} \mathrm{~d} V=\int_{S} \bar{t}_{i} \delta u_{i} \mathrm{~d} S \quad \text { with } i, j=1, \ldots, 3
$$


$V$ is the body volume and $S$ the external surface of the volume where stress is restricted as $t_{i}=\bar{t}_{i}$. As external charges are not considered in the present paper, principle (21) reduces to

$$
\int_{V} \sigma_{i j} \delta \varepsilon_{i j} \mathrm{~d} V=0 \quad \text { with } i, j=1, \ldots, 3
$$

The metal matrix composite is discretized in hexahedral three-dimensional finite elements with eight nodes and eight integration points. The volumetric part of the displacement gradient is interpolated with a reduced selective scheme [27, 28], in order to avoid the development of eigenstrain modes. The finite element discretization of Equation (22), for a generic time increment $n$, leads to a standard linear algebraic system of equations:

$$
\mathbf{K}_{n} \Delta \mathbf{u}_{n}=\Delta \mathbf{f}_{n}
$$

where $\Delta \mathbf{u}_{n}$ is the displacement increment vector, $\mathbf{K}_{n}$ is the global stiffness matrix and $\Delta \mathbf{f}_{n}$ is the incremental nodal force vector, all evaluated at time $t_{n}$ and corresponding to time increment $\Delta t$. The solution of system (23) is then used to update the configuration and all the state variables.

\subsection{Time increment optimization}

It is known that the forward gradient time integration procedure described above can become inaccurate in situations where the plastic flow rate changes very rapidly [29]. However, the implementation of an automatic time-step optimization algorithm can account for these changes. The automatic time-step algorithm proposed is based on a measure of the rate of change of the plastic flow rate tensor. The automatic optimization algorithm uses two-step reduction criteria. In the first one a control parameter $C_{\max }$ is calculated. $C_{\max }$ is a scalar that represents the maximum absolute value, over all the integration points (NGP) of the difference between the equivalent plastic strain rate $\dot{\bar{\varepsilon}}^{\mathrm{p}}$, at the end and beginning of the current increment, multiplied by the size of the time increment itself:

$$
C_{\max }=\max _{\mathrm{NGP}}\left|\dot{\bar{\varepsilon}}_{n+1}^{\mathrm{p}}-\dot{\bar{\varepsilon}}_{n}^{\mathrm{p}}\right| \Delta t
$$

In this criterion $C_{\max }$ is compared with a prescribed tolerance $C_{\mathrm{tol}}$ defined as

$$
C_{\mathrm{tol}}=\delta \frac{s_{0}}{E_{M}}
$$

where $E_{\mathrm{M}}$ is the Young's modulus of the matrix material, $s_{0}$ is the initial value of parameter $s$ and $\delta \in[0 ; 1]$.

The second criteria is based on the determination of $D_{\max }$, the maximum value of the increment of the equivalent plastic strain over all the integration points of the structure:

$$
D_{\max }=\max _{\mathrm{NGP}} \Delta \overline{\bar{\varepsilon}}^{\mathrm{p}}
$$

This value is also compared with a prescribed tolerance $D_{\text {tol }}$.

A time-step reduction factor $R$ is calculated after the determination of the two optimization parameters $C_{\max }$ and $D_{\max }$ by combining the two criteria,

$$
R=\max \left(\frac{C_{\max }}{C_{\mathrm{tol}}}, \frac{D_{\max }}{D_{\mathrm{tol}}}\right)
$$


Table I. Algorithm for the time integration procedure.

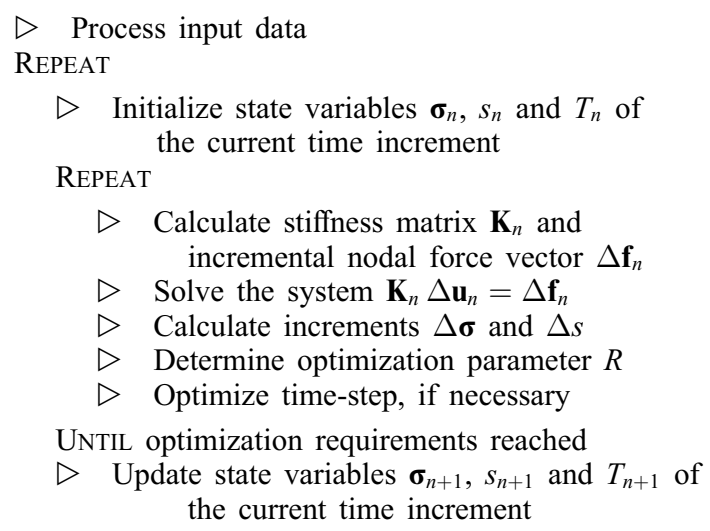

If $R>1$ than the time step must be reduced with

$$
\Delta t_{\text {new }} \leadsto \frac{R_{\text {cut }}}{R} \Delta t_{\text {old }}
$$

where $R_{\text {cut }}$ is an optimization reduction factor. If $R<1$ the time step does not need to be cut and the next step can even be increased. May this be the case and the following step is enlarged with a factor $1.1 \leqslant R_{\mathrm{enl}} \leqslant 2.5[10]$

$$
\Delta t_{\text {new }} \leadsto R_{\text {enl }} \Delta t_{\text {old }}
$$

depending on the magnitude of $R$.

The enlargement factor, $R_{\text {enl }}$, can be calculated dynamically for each time step according to the expression described below, in analogy with the iteration schemes proposed by Patankar [30]:

$$
R_{\mathrm{enl}}=2.5-1.4\left\{\frac{\exp \left(F_{\mathrm{c}}(R-0.2) / 0.6\right)-1}{\exp \left(F_{\mathrm{c}}\right)-1}\right\}
$$

where $F_{\mathrm{c}}$ is a correction factor defining the shape of the optimization profile.

\subsection{Global algorithm}

The generic algorithm of the procedure described above can be synthesized as in Table I. This algorithm was implemented in the finite element code TROTE3D which is devoted to the numerical calculation of thermally induced residual stresses in MMCs.

\section{RESULTS AND DISCUSSION}

A large number of numerical simulations was performed to test the numerical efficiency of the proposed algorithms. The simulations were performed with the meshes represented in Figures 1 and 2. The mesh on Figure 1 corresponds to a representative cell for a continuous fibre-reinforced 


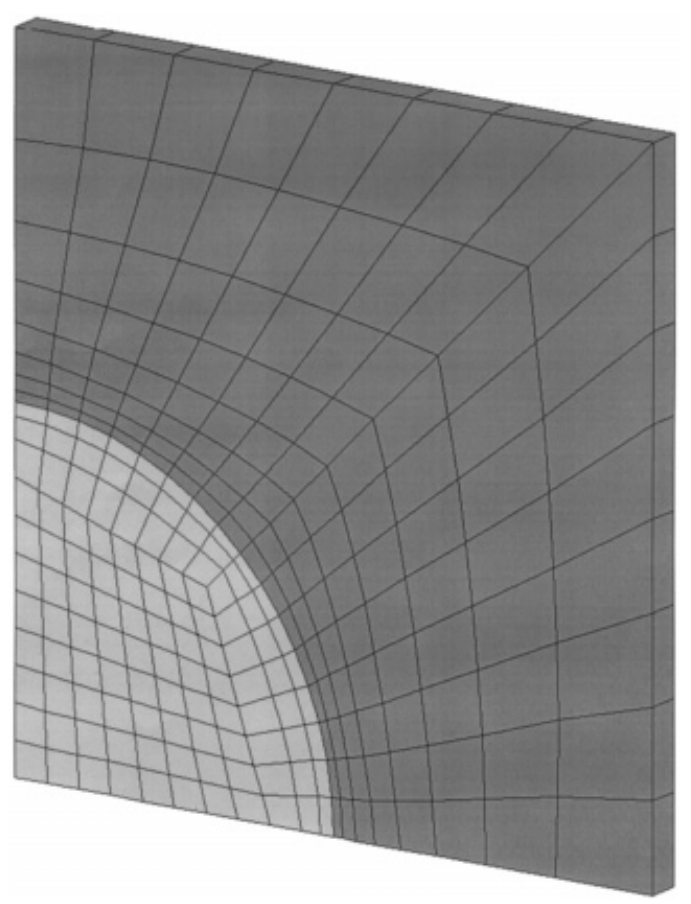

Figure 1. MESH 1, representative of a continuously reinforced metal matrix, composite with 240 elements and 536 nodes.

metal matrix composite and, in Figure 2, is a representative cell for a cylindrical particle reinforced MMC.

The boundary conditions specified were such that coordinate planes $O x y, O x z$, and $O y z$ were planes of symmetry, leaving the other three planes as free moving.

All the simulations with the meshes described above were performed with Al-SiC composites with 20 per cent volume fraction of reinforcement material. The material properties (Young's modulus, Lamé coefficient and CTE - coefficient of thermal expansion) for the aluminium matrix are temperature dependent and given as [9]

$$
\begin{aligned}
E_{\mathrm{M}}(T) & =73474-43.48 \times(T-273)(\mathrm{MPa}) \\
\mu_{\mathrm{M}}(T) & =27041-17.057 \times(T-273)(\mathrm{MPa}) \\
\alpha_{\mathrm{M}} & =0.287 \times 10^{-4} \mathrm{~K}^{-1}
\end{aligned}
$$

and for the reinforcement material:

$$
\begin{aligned}
& E_{\mathrm{R}}=41 \times 10^{4} \mathrm{MPa} \\
& \mu_{\mathrm{R}}=16.532 \times 10^{4} \mathrm{MPa} \\
& \alpha_{\mathrm{R}}=0.43 \times 10^{-5} \mathrm{~K}^{-1}
\end{aligned}
$$




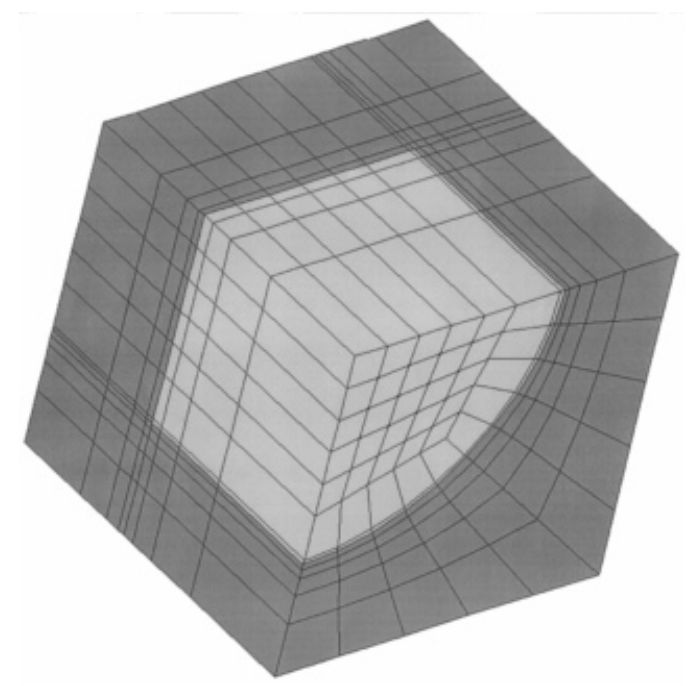

Figure 2. MESH 2, representative of a cylindrical particle reinforced metal matrix composite with 760 elements and 1017 nodes.

Table II. Material parameters for the costitutive functions.

\begin{tabular}{lccc}
\hline Parameter & Value & Parameter & Value \\
\hline$A$ & $1.91 \times 10^{7} \mathrm{~s}^{-1}$ & $a$ & 1.3 \\
$Q / R_{\mathrm{g}}$ & $21090 \mathrm{~K}$ & $\bar{s}$ & $18.9 \mathrm{MPa}$ \\
$\xi$ & 7 & $s_{0}$ & $18 \mathrm{MPa}$ \\
$m$ & 0.23348 & $n$ & 0.07049 \\
$h_{0}$ & $1115.6 \mathrm{MPa}$ & & \\
\hline
\end{tabular}

The material parameters used in the constitutive Equations (6)-(8) were chosen after Brown et al. [22] and are listed in Table II.

A constant rate cooling down process was considered with the initial and final temperatures being $T_{\mathrm{ini}}=933 \mathrm{~K}$ and $T_{\text {end }}=293 \mathrm{~K}$, respectively, and with the cooling rate $\dot{T}=-100 \mathrm{~K} \mathrm{~s}^{-1}$.

\subsection{Accurate results}

For comparison purposes an accurate reference solution was obtained using a very small constant time increment, $\mathrm{d} t=0.001 \mathrm{~s}$, and a value of $\Phi=0.65$. The accurate temperature evolution of the equivalent stress $\bar{\sigma}$, parameter $s$ and equivalent plastic strain rate $\dot{\bar{\varepsilon}}^{\mathrm{p}}$ is shown in Figures 3 and 4 for both meshes considered. These results were obtained on an integration point in the matrix material, close to the matrix/reinforcement interface. The final plastic strain rate fields can be visualized in Figures 5 and 6 . These results are equivalent to the unidimensional simulations of Teodosiu and Menezes [10] and Suéry et al. [9] and the plastic strain rate field in Mesh 2 (Figure 6) is identical to the one obtained by Haddadi [31]. 


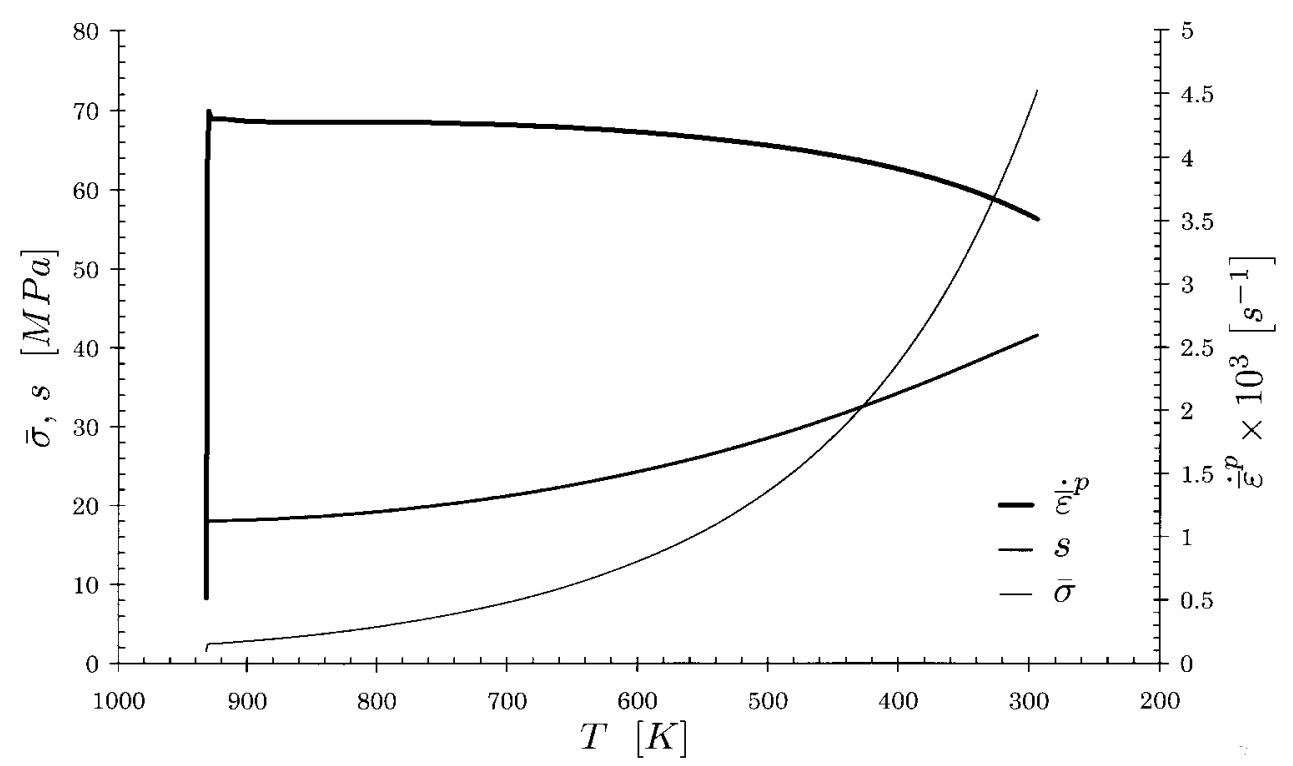

Figure 3. Accurate results for MESH 1. Equivalent stress $\bar{\sigma}$, parameter $s$ and plastic strain rate $\dot{\bar{\varepsilon}}^{\mathrm{p}}$.

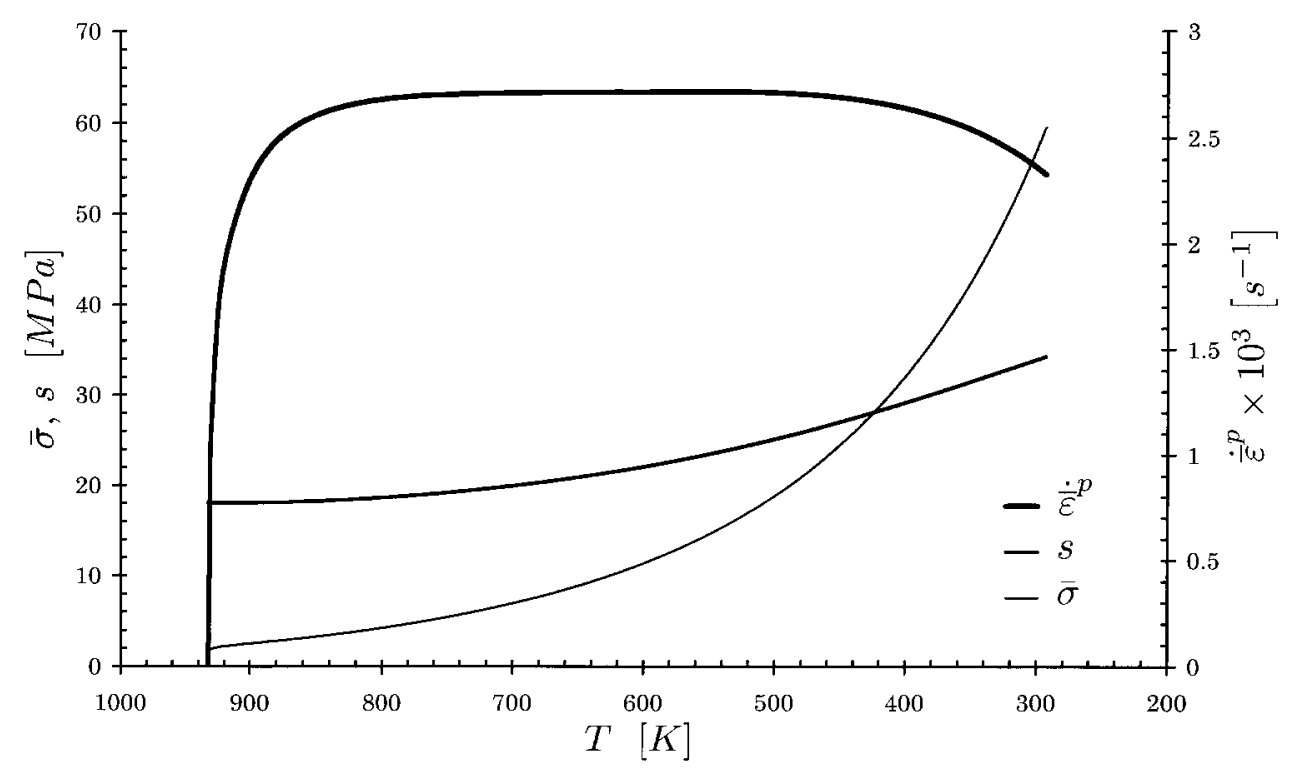

Figure 4. Accurate results for MESH 2. Equivalent stress $\bar{\sigma}$, parameter $s$ and plastic strain rate $\dot{\bar{\varepsilon}}^{\mathrm{p}}$. 


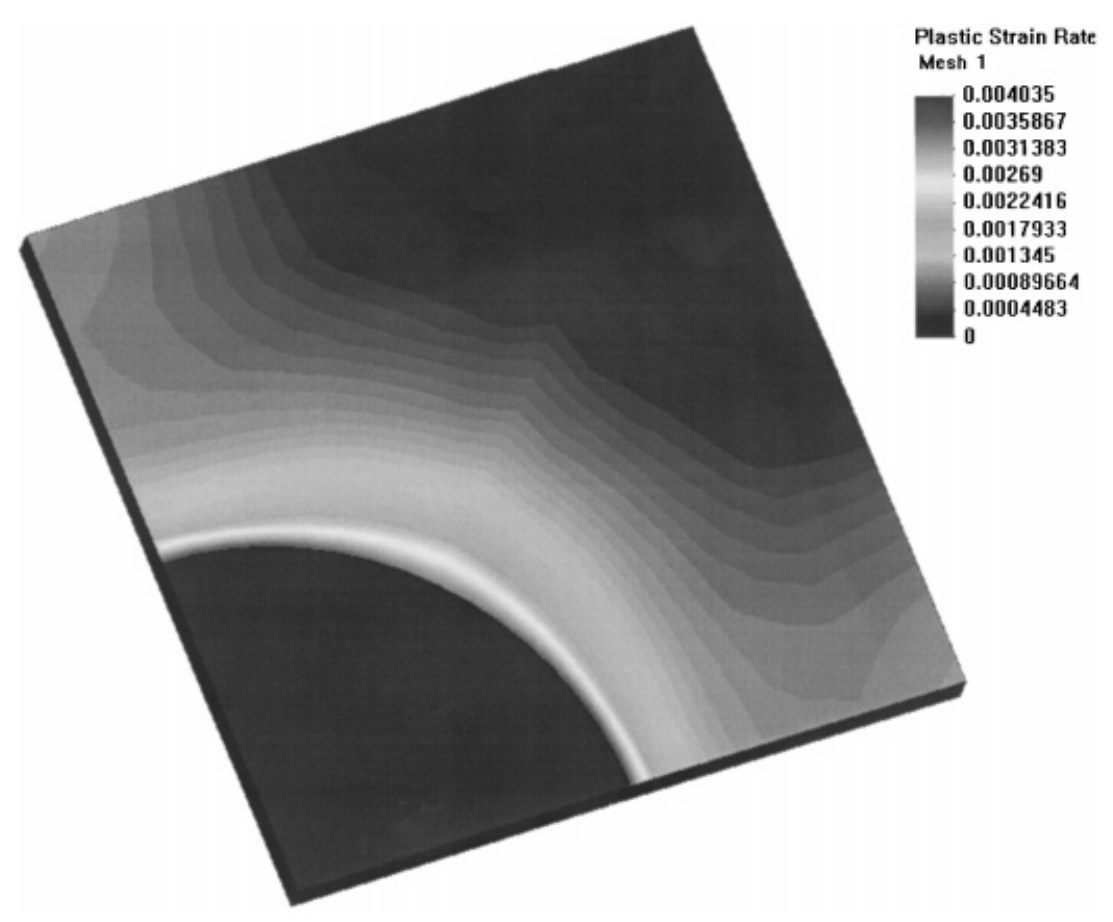

Figure 5. Final plastic strain rate field for the continuously reinforced MMC (MESH 1).

\subsection{Optimized simulations}

Further calculations were performed in order to evaluate the performance and accuracy of the algorithms proposed, for a set of values of the parameters $\Phi, \delta$ and the initial time step $\mathrm{d} t_{\text {ini }}$. The optimization reduction factor $R_{\text {cut }}$ was assumed to be equal to 0.85 after the studies performed by Teodosiu and Menezes [10]. The results obtained were then compared with the accurate solutions. The influence of the numerical parameters $\Phi, \delta$ and $\mathrm{d} t_{\text {ini }}$ on the total CPU time and on the quality of the results was evaluated. This quality was measured calculating the relative errors of the final values of $s, \bar{\sigma}$ and $\dot{\bar{\varepsilon}}$. Figures $7-9$ show the evolution of the relative errors of $s, \bar{\sigma}$ and $\dot{\bar{\varepsilon}}$ p with $\Phi, \delta$ and the initial time step $\mathrm{d} t_{\mathrm{ini}}$, respectively, and for both meshes.

For the range of values tested no significant errors are observed in either $s$ or $\bar{\sigma}$. Nevertheless, quite large errors develop eventually in the plastic strain rate $\dot{\bar{\varepsilon}}^{\mathrm{p}}$. This can be explained by the results shown in Figure 10, which shows the evolution of the plastic strain rate $\dot{\bar{\varepsilon}}^{\text {p }}$ with the temperature, for three different values of $\delta$ and for Mesh 1. The accurate result for Mesh 1 is also added for comparison purposes. The fluctuations of $\dot{\bar{\varepsilon}}$ p that increase with $\delta$, are directely associated with the increase of the time step. This fact confirms that large time steps lead to large variations of the plastic strain rate that deteriorate the stability of the forward gradient time integration procedure. The implementation of the proposed time-step control algorithm avoids the possibility of a numerical crash. The final solution can be obtained even with severe oscilations on the evolution of the plastic strain rate $\dot{\bar{\varepsilon}}^{\mathrm{p}}$. The value of $\Phi$ is also a determinant factor for the accuracy of the results. For low values of $\Phi(\Phi \leqslant 0.40)$ the solution cannot be calculated, independently 


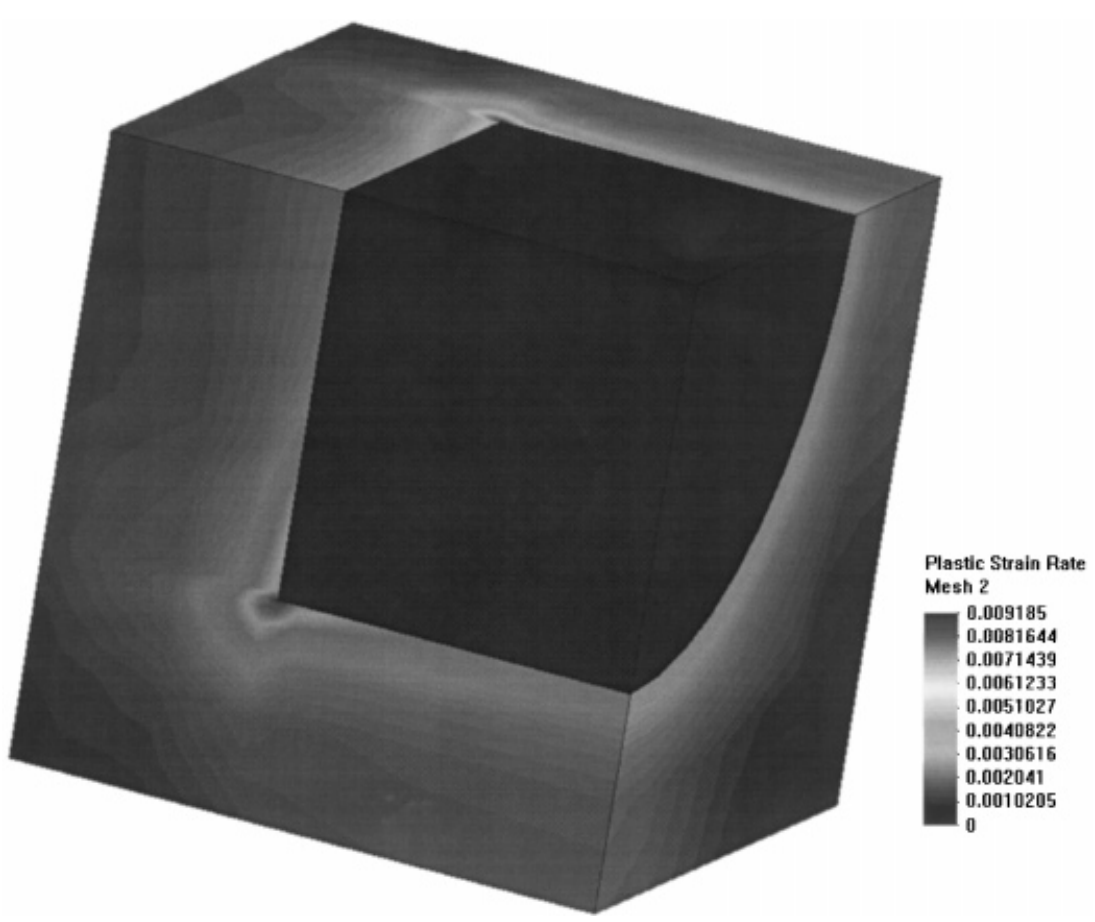

Figure 6. Final plastic strain rate field for the cylindrical particle-reinforced MMC (MESH 2).

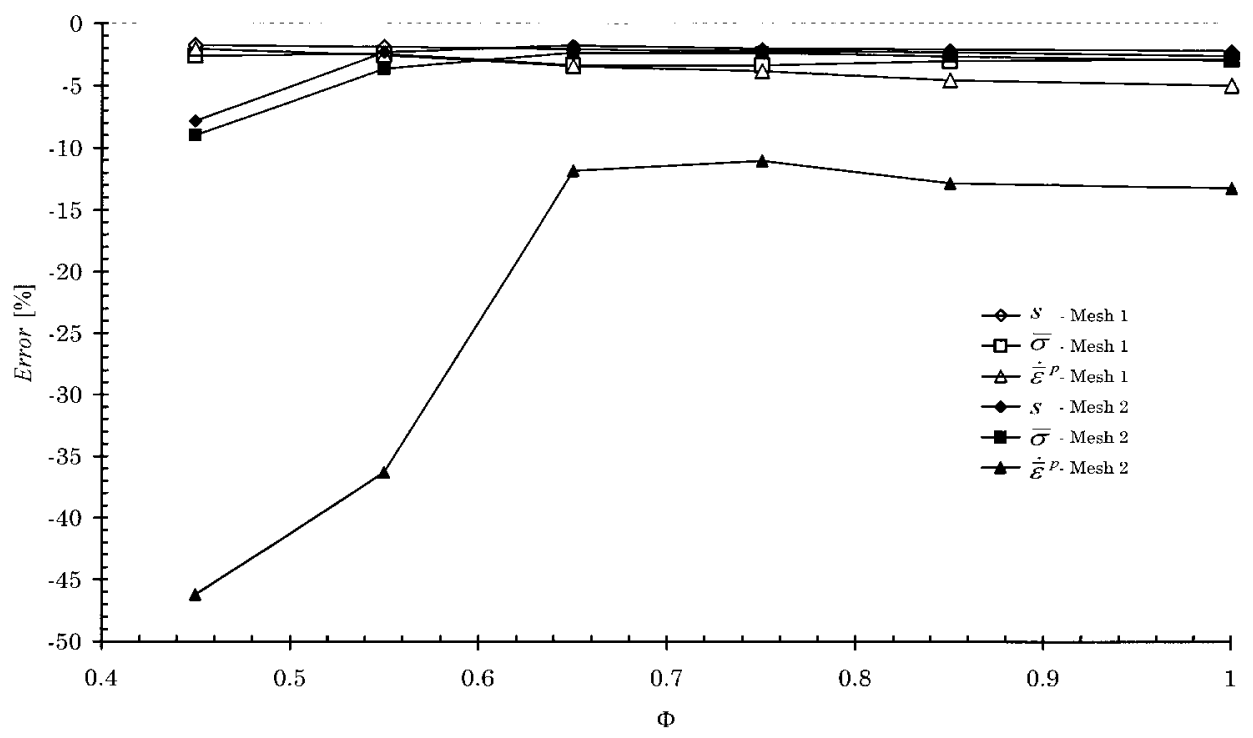

Figure 7. Errors obtained in the optimized numerical simulations as a function of $\Phi\left(\delta=0.1, \mathrm{~d} t_{\text {ini }}=0.001\right)$. 


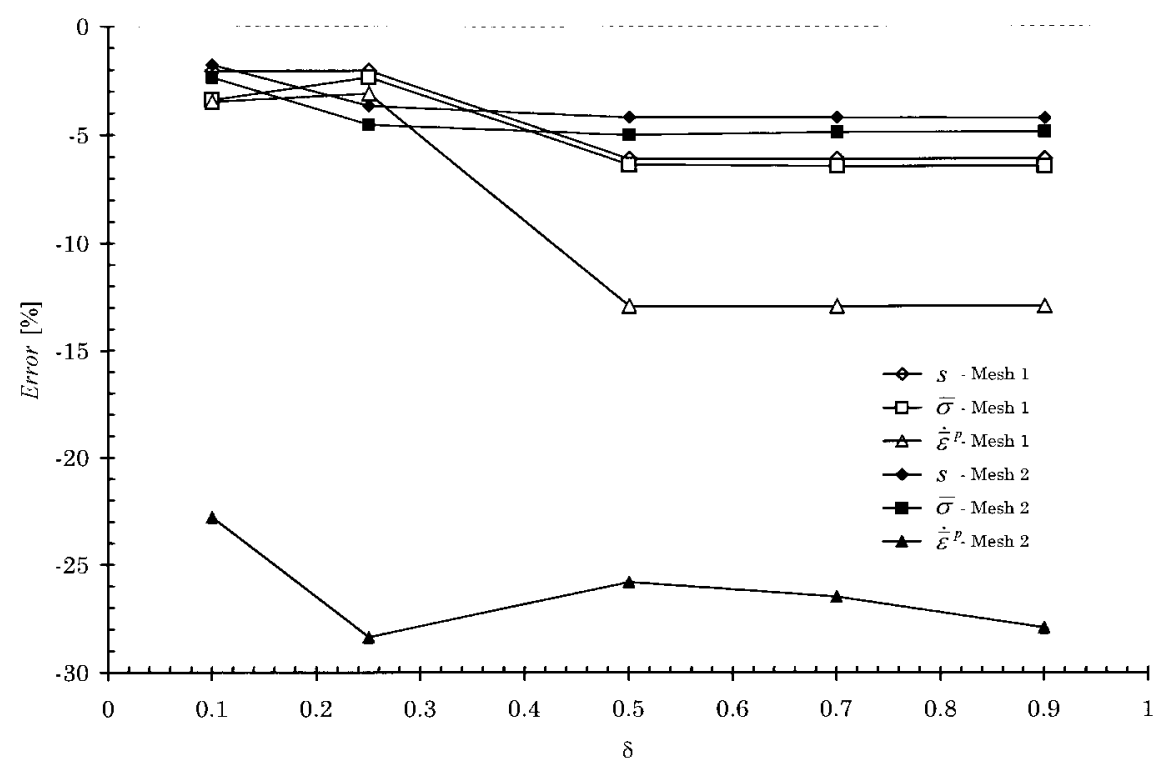

Figure 8. Errors obtained in the optimized numerical simulations as a function of $\delta\left(\Phi=0.65, \mathrm{~d} t_{\text {ini }}=0.001\right)$.

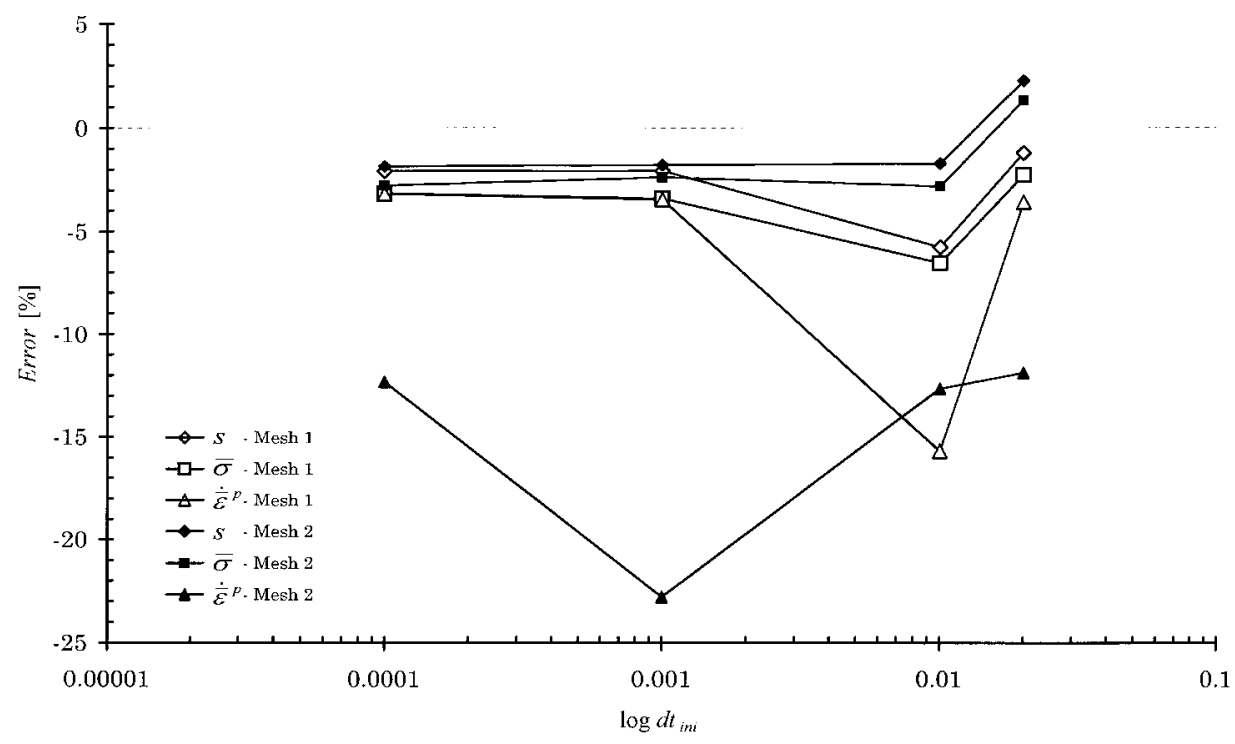

Figure 9. Errors obtained in the optimized numerical simulations as a function of $\mathrm{d} t_{\text {ini }}(\Phi=0.65, \delta=0.1)$. 


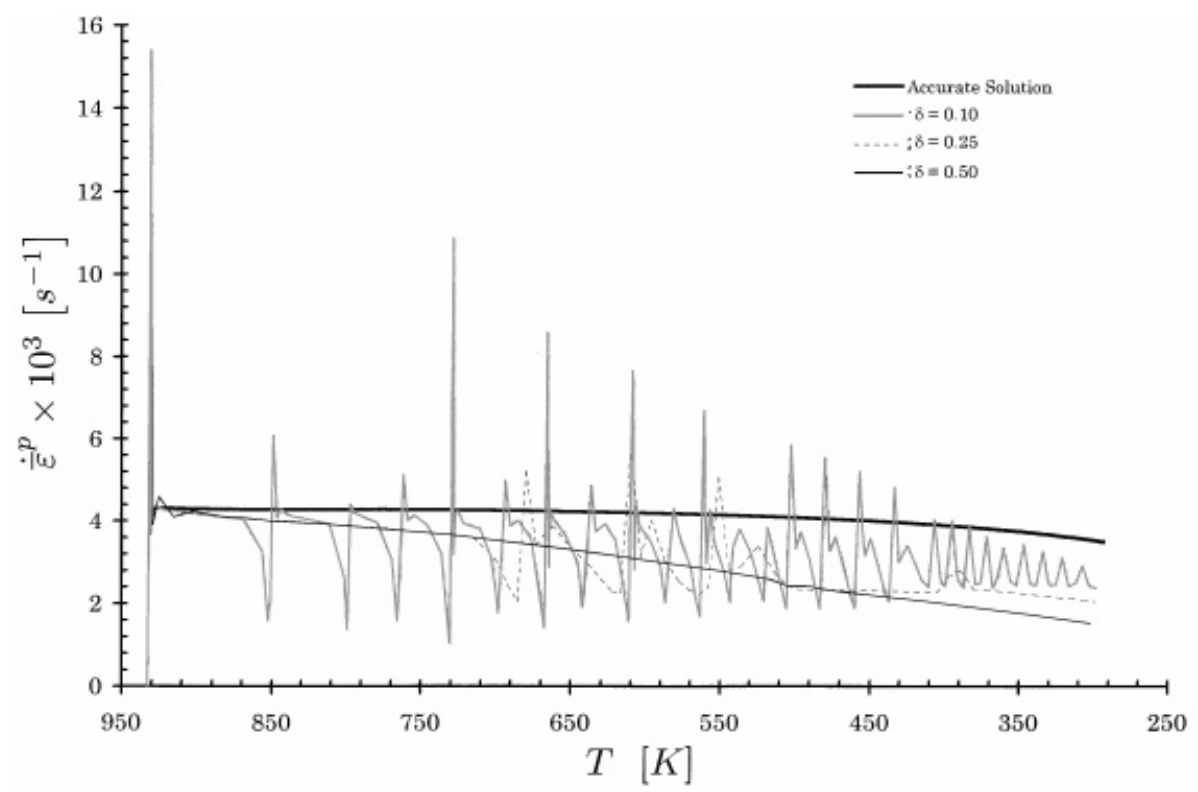

Figure 10. Temperature evolution of the equivalent plastic strain rate $\dot{\bar{\varepsilon}}^{\mathrm{p}}$ for $\delta=0.1,0.25$ and 0.5 .

of the value of $\delta$ used. In fact, when a small value of $\Phi$ is adopted, the algorithm aproaches a pure explicit time integration method, where the high non-linearities of the constitutive equations strongly limitate the size of the time step. The influence of the parameter $\mathrm{d} t_{\text {ini }}$ on the accuracy of the final results is much lower than the other control parameters $(\Phi$ and $\delta$ ) and the automatic control of the time increment size rapidly eliminates its influence during the first steps of the simulation. This fact leads to the conclusion that $\delta$ works as an accuracy control parameter. Thus, it is clearly more economical in terms of CPU to use larger values of $\delta$. However, increasing $\delta$ leads to higher variations in the equivalent plastic strain rate. These fluctuations are clearly undesirable and a compromise value for $\delta$ should be chosen.

The total CPU times required by the accurate solutions was $\mathrm{CPU}_{\mathrm{acc}}^{1}=35977$ and $\mathrm{CPU}_{\mathrm{acc}}^{2}=$ $150388 \mathrm{~s}$ for Mesh 1 and Mesh 2, respectively. These values were obtained with a $166 \mathrm{MHz}$ Intel $^{\mathrm{TM}}$ Pentium ${ }^{\mathrm{TM}}$ II personal computer with 128 Mbytes of RAM memory. The numerical control parameters have some degree of effect on the relative CPU time. The relative CPU times are shown in Figure 11 as a function of $\Phi$ and $\delta$, for Mesh 1. Identical behaviour is verified for Mesh 2 and no significant evolution of the relative CPU time, calculated as $\mathrm{CPU}_{\text {rel }}=\mathrm{CPU} / \mathrm{CPU}_{\text {acc }}$, was detected as a function of $\mathrm{d} t_{\text {ini }}$.

For values of $\Phi<0.6$ the computational time increases significantly, as can be seen in Figure 11. In fact, it is in these situations that the numerical instabilities referred above appear (see Figure 7) and the automatic time-step control leads to very small time steps and thus to the verified increase in the overall CPU time. As seen clearly in Figure 10, decreasing $\delta$ leads to much better results but also to higher CPU times (see Figure 11). It should be noted that the implementation of the proposed automatic time-step control algorithm leads to a drastic reduction (more than 


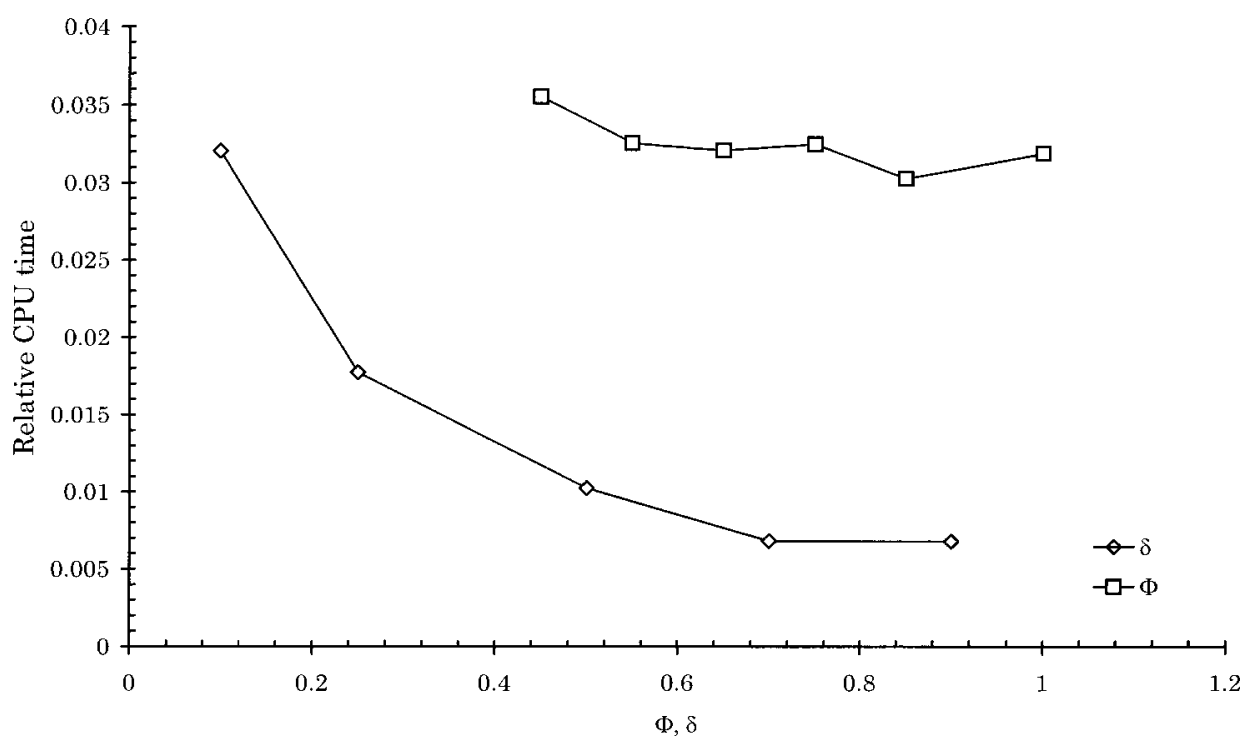

Figure 11. Relative CPU times as a function of $\Phi$ and $\delta$ for MESH 1.

90 per cent) of the CPU time, without significant changes in the numerial results, when compared to the accurate solutions, obtained with a constant time step.

\section{CONCLUSIONS}

A three-dimensional mechanical model applicable to metal matrix composites was presented. The mechanical model considers the reinforcement component to have a thermoelastic behaviour and the matrix material to behave thermo-elastoviscoplastically. The model was applied in a finite element method algorithm and tested with some numerical examples. Residual stresses and deformation fields were determined in $\mathrm{Al}-\mathrm{SiC}$ composites with 20 per cent volume fraction of reinforcement material. Two meshes were used in order to represent a continuously reinforced and a cylindrical reinforcement metal matrix composite. The numerical parameters that control the algorithms were studied. The choice of the numerical values for the parameters $\delta, \Phi$ and the initial time step $\mathrm{d} t_{\text {ini }}$ must lead to a compromise between accuracy and computational cost. Based upon the presented calculations, the values $\delta=0.1, \Phi=0.65$ and $\mathrm{d} t_{\text {ini }}=0.001$, seem reasonable.

\section{ACKNOWLEDGEMENTS}

The authors would like to acknowledge the financial support given to CEMUC by the FCT (Portuguese Science and Technology Foundation).

\section{REFERENCES}

1. Povirk GL, Needlemann A, Nutt SR. An analysis of residual stress formation in whisker-reinforced Al-SiC composites. Materials Science and Engineering A 1990; 125:129. 
2. Povirk GL, Needlemann A, Nutt SR. An analysis of the effect of residual stresses on deformation and damage mechanisms in Al-SiC composites. Materials Science and Engineering A 1991; 132:31.

3. Shen Y-L, Needlemann A, Suresh S. Coefficients of thermal expansion of metal-matrix composites for electronic packaging. Metallurgical Transactions A 1994; 25A:839.

4. Shaw LL, Miracle DB. Effects of an interfacial region on the transverse behaviour of metal matrix composites-A finite element analysis. Acta Materialia 1996; 44(5):2043.

5. Ward PJ, Atkinson HV, Anderson PRG, Elias LG, Garcia B, Kahlen L, Rodriguez-Ibabe J-M. ASDASD. Acta Materialia 1996; 44(5):1717.

6. Michel JC, Suquet P. An analytical and numerical study of the overall behaviour of metal-matrix composites. Modelling and Simulation in Materials Science and Engineering 1994; 2:637.

7. Ramakrishnan N. An analytical study on strengthening of particulate reinforced metal matrix composites. Acta Materialia 1996; 44(1):69.

8. Zahl DB, Schmauder S, McMeeking RM. Mechanical behaviour of residual stressed composites with ductile and brittle constituents. Modelling and Simulation in Materials Science and Engineering 1994; 2:267.

9. Suéry M, Teodosiu C, Menezes LF. Thermal residual stresses in plastic-reinforced viscoplastic metal matrix composites. Materials Science and Engineering A 1993; 167:97.

10. Teodosiu C, Menezes LF. Numerical methods for calculating thermal residual stresses in particle reinforced metal matrix composites. Revue Roumaine Sciences Techniques-Mécanique Appliquée 1991; 36(3,4):243.

11. Kolhe R, Hui CY, Ustundag E, Sass SL. Residual thermal stresses and calculation of the critical metal particle size for interfacial crack extension in metal-ceramic matrix composites. Acta Materialia 1996; 44(1):279.

12. Zywicz E, Parks DM. Thermo-viscoplastic residual stresses in metal matrix composites. Computer Science and Technology 1998; 33:295.

13. Vedula M, Pangborn RN, Queeney RA. Modification of residual thermal stress in a metal-matrix composite with the use of a taylored interfacial region. Composites 1988; 19(2):133.

14. Ledbetter HM, Austin MW. Internal strain (stress) in SiC-Al particle-reinforced composite. Materials Science and Engineering $A$ 1987; 89:53.

15. Ho S, Saigal A. Thermal residual stresses and mechanical behaviour of cast SiC/Al. Materials Science and Engineering A 1994; 183:39.

16. Arsenault RJ, Taya M. Thermal residual stress in metal matrix composite. Acta Metallurgica 1987; 35(3):651.

17. Jain M, MacEwen SR, Wu L. Finite element modelling of residual stresses and strength differential effect in discontinuously reinforced metal matrix composites. Materials Science and Engineering A 1994; 183:111.

18. Withers PJ, Stobbs WM, Pedersen OB. The application of the Eshelby method of internal stress determination to short fibre metal matrix composites. Acta Metallurgica 1989; 37(11):3061.

19. Dunn ML, Taya M. Elastic-plastic thermal stresses and deformation of short-fibre composites. Journal of Materials Science 1994; 29:2053.

20. Miller AK, Sherby OD. A simplified phenomenological model for non-elastic deformation: Prediction of pure aluminum behaviour and incorporation of solute strengthening effects. Acta Metallurgica 1978; 26:289.

21. Anand L. Constitutive equations for hot-working of metals. International Journal of Plasticity 1985; 1:213.

22. Brown SB, Kwon HK, Anand L. An internal variable constitutive model for hot working of metals. International Journal of Plasticity 1989; 5:95.

23. Lush A, Anand L. Implicit time-integration procedures for a set of internal variable constitutive equations for hotworking. Proceedings of the NUMIFORM'86 1986; 131.

24. Teixeira-Dias F, Menezes LF. Modelling and three-dimensional numerical simulation of residual stresses in metal matrix composites. Proceedings of the AMPT'97-Advances in Materials and Processing Technologies 1997; 1:68.

25. Teixeira-Dias F, Menezes LF. Modelling and numerical simulation of residual stresses in metal matrix composites. Proceedings of the ICCE/6 - 6th International Conference on Composites Engineering 1999; 177.

26. Zienkiewicz OC, Taylor RL. The Finite Element Method (4th edn) vol. 1. McGraw-Hill Book Company Europe, Berkshire, England 1994; 225.

27. Hughes TJR. Generalization of selective integration procedures to anisotropic and non-linear media. International Journal for Numerical Methods in Engineering 1980; 15:1413.

28. Shimodarira H. Equivalence between mixed models and displacement models using reduced integration. International Journal for Numerical Methods in Engineering 1985; 21:89.

29. Anand L, Lush A, Briceno MF, Parks DM. A time integration procedure for a set of internal variable type elastoviscoplastic constitutive equations. Research Report-Department of Mechanical Engineering, MIT, 1985.

30. Patankar SV, Numerical Heat Transfer and Fluid Flow. Hemisphere/McGraw-Hill: Washington, DC, 1980.

31. Haddadi H. Ph.D. Thesis, Matériaux composites à Matrice Métallique: Modélisation du Comportement thermoélastoviscoplastique et Résolution de Problèmes aux Limites 3D par la Méthode des Éléments Finis, LPMTM Université Paris-Nord, 1996. 